\title{
Epigenome-Wide Study Identified Methylation Sites Associated with the Risk of Obesity
}

\author{
Majid Nikpay ${ }^{1, *}$, Sepehr Ravati ${ }^{2}$, Robert Dent ${ }^{3}$ (D) and Ruth McPherson ${ }^{1,4, *}$ \\ 1 Ruddy Canadian Cardiovascular Genetics Centre, University of Ottawa Heart Institute, 40 Ruskin St-H4208, \\ Ottawa, ON K1Y 4W7, Canada \\ 2 Plastenor Technologies Company, Montreal, QC H2P 2G4, Canada; ravatisepehr@gmail.com \\ 3 Department of Medicine, Division of Endocrinology, University of Ottawa, the Ottawa Hospital, \\ Ottawa, ON K1Y 4E9, Canada; bdent@toh.ca \\ 4 Atherogenomics Laboratory, University of Ottawa Heart Institute, Ottawa, ON K1Y 4W7, Canada \\ * Correspondence: mnikpay@ottawaheart.ca (M.N.); rmcpherson@ottawaheart.ca (R.M.)
}

Citation: Nikpay, M.; Ravati, S.;

Dent, R.; McPherson, R.

Epigenome-Wide Study Identified Methylation Sites Associated with the Risk of Obesity. Nutrients 2021, 13, 1984. https://doi.org/10.3390/ nu13061984

Academic Editor: M. Luisa Bonet

Received: 5 May 2021

Accepted: 7 June 2021

Published: 9 June 2021

Publisher's Note: MDPI stays neutral with regard to jurisdictional claims in published maps and institutional affiliations.

Copyright: (C) 2021 by the authors. Licensee MDPI, Basel, Switzerland. This article is an open access article distributed under the terms and conditions of the Creative Commons Attribution (CC BY) license (https:// creativecommons.org/licenses/by/ $4.0 /)$.

\begin{abstract}
Here, we performed a genome-wide search for methylation sites that contribute to the risk of obesity. We integrated methylation quantitative trait locus (mQTL) data with BMI GWAS information through a SNP-based multiomics approach to identify genomic regions where mQTLs for a methylation site co-localize with obesity risk SNPs. We then tested whether the identified site contributed to BMI through Mendelian randomization. We identified multiple methylation sites causally contributing to the risk of obesity. We validated these findings through a replication stage. By integrating expression quantitative trait locus (eQTL) data, we noted that lower methylation at cg21178254 site upstream of CCNL1 contributes to obesity by increasing the expression of this gene. Higher methylation at cg02814054 increases the risk of obesity by lowering the expression of MAST3, whereas lower methylation at cg06028605 contributes to obesity by decreasing the expression of SLC5A11. Finally, we noted that rare variants within 2p23.3 impact obesity by making the cg01884057 site more susceptible to methylation, which consequently lowers the expression of $P O M C, A D C Y 3$ and DNAJC27. In this study, we identify methylation sites associated with the risk of obesity and reveal the mechanism whereby a number of these sites exert their effects. This study provides a framework to perform an omics-wide association study for a phenotype and to understand the mechanism whereby a rare variant causes a disease.
\end{abstract}

Keywords: obesity; EWAS; epigenetics; multiomics; Mendelian randomization

\section{Introduction}

Obesity is a complex phenotype and the outcome of numerous genes and environmental factors. Epigenetic sites are considered as the sites of gene-environment interactions. Epigenetics provides an elegant solution to modify gene expression activity in response to external stimuli without altering the DNA code. As such, it has an important role in the regulation and manifestation of complex phenotypes.

The relation between epigenetics and obesity has been the subject of numerous studies over the past few years [1,2]. Methodological improvements, and the global increase in obesity, have contributed to this interest. The general consensus from these studies is that interindividual variation in epigenetic modifications correlates with body weight. Furthermore, findings from these studies support not only a role for epigenetics in gaining weight, but also epigenetic alterations as a response to obesity [1-6].

As reviewed by Ling et al. [1], the majority of previous studies had small sample sizes, which lowers the power of statistical tests. Furthermore, they typically measured both epigenetic levels and BMI in the same group of individuals and then investigated the epigenetic sites that showed differential levels of modifications in individuals with a higher BMI than those with a lower BMI; however, such a design cannot tell us whether a 
significant association indicates causation (e.g., Methylation at a site $\rightarrow$ Obesity), correlation (Methylation $\leftarrow$ confounders $\rightarrow$ Obesity) or reverse causation (Obesity $\rightarrow$ Methylation).

To overcome these issues, in this study, we used Mendelian randomization (MR) that can control for both confounding and reverse causation. MR is a form of instrumental variable analysis that investigates the relationship between the exposure (DNA methylation) and the outcome (BMI) using an instrument (a set of independent SNPs) that is known to cause change in the exposure. Alleles of independent SNPs are randomly allocated to offspring at conception (Mendel's second law); therefore, an instrument of SNPs is inherently immune to the confounding effect of environmental factors that can bias an association study. Furthermore, by excluding SNPs with pleiotropic effect (Methylation $\leftarrow$ $\mathrm{SNP} \rightarrow$ Obesity) from the instrument, it is possible to rule out the correlation scenario, and by swapping the places of exposure and outcome and repeating the test, we can investigate the possibility of reverse causation.

To increase the statistical power of our analyses, in this study, we used a two-sample MR design (Figure 1) that incorporated data from separate studies to estimate a causal effect of the exposure on the outcome. Hence, with this design, we can achieve a better statistical power by including data from the GWAS consortia [7].

Examining the association between change in DNA methylation at every site in the genome and obesity using MR is cumbersome. Therefore, in this study, we used a SNPbased multiomics pipeline that also included MR, in order to efficiently narrow down our search and identify methylation sites that contribute to the risk of obesity. We validated our findings through the replication stage; we also investigated the mechanism whereby these sites contribute to obesity by integrating eQTLs data into our analysis.

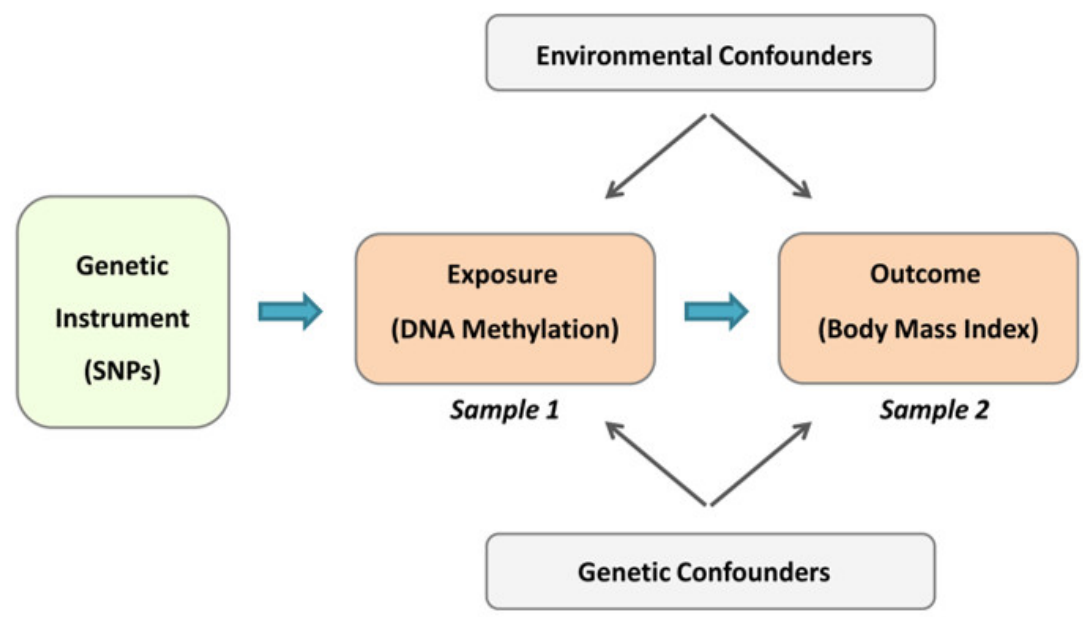

Figure 1. Concept of two-sample Mendelian randomization (MR) design. Under this design, we first constructed an instrument based on a set of independent SNPs that were significantly associated with the exposure in the first sample. Next, we contrasted effect sizes (betas) of SNPs on the exposure with their corresponding effect sizes on the outcome (obtained from the second sample) to find out if there was a significant association. This design is immune to confounding environmental factors because it uses SNPs as instrument. Furthermore, by removing SNPs with peliotropic effect, genetic confounding is prevented. Further information is provided in the Methods section.

\section{Methods}

Our SNP-based multiomics design was previously described [8,9]. In summary, our approach requires full GWAS summary statistics as input files and a genotype file to calculate the linkage disequilibrium (LD) between SNPs. In this study, we used mQTL data from McRae et al. [10] and GWAS data for BMI from the meta-analysis of the UK Biobank (UKBB) and Giant consortium [11] to identify methylation sites that contribute to 
the risk of obesity. We used the genotype data from the 1000 genomes $(n=503$ of European ancestry) to calculate the LD between SNPs.

Initially, we tested whether SNPs that are associated with BMI co-localize with mQTLs for a methylation site. Co-localization analysis was done using the SMR software (version 1.03) [12] that can also differentiate between a pleiotropic effect (its null hypothesis) and a linkage effect through the HEIDI test. Methylation sites that their QTLs co-localize with BMI risk SNPs (with $\mathrm{P}_{\mathrm{SMR}}<5 \times 10^{-8}$ and $\mathrm{P}_{\mathrm{HEIDI}}>0.05$ ), were then subjected to Mendelian randomization analysis. Note that $\mathrm{P}_{\mathrm{SMR}}$ indicates the $p$-value from the co-localization test and $\mathrm{P}_{\mathrm{HEIDI}}$ represents the $p$-value from the pleiotropy test. Mendelian randomization (MR) aims to investigate the relationship between the exposure (methylation at a site) and the outcome (BMI) using a set of independent SNPs (instrument). SNPs included in the instrument must meet a number of criteria.

(1) They must not be in LD. In this study, we used SNPs that were in linkage equilibrium $\left(\mathrm{r}^{2}<0.05\right)$.

(2) They must not show a pleiotropic effect (i.e., Exposure $\leftarrow$ SNP $\rightarrow$ Outcome). We excluded such SNPs from the instrument by using $\left(\mathrm{P}_{\text {HEIDI }}<0.01\right)$.

(3) They must be significantly associated with exposure; we used SNPs that are associated with exposure at the GWAS significance level $\left(p<5 \times 10^{-8}\right)$.

We did the MR analysis using the GSMR algorithm implemented in GCTA software (version 1.92) [13]. As compared to other methods for MR analysis, GSMR automatically detects and removes SNPs that have a pleiotropic effect on both exposure and outcome; in addition, it accounts for the sampling variance in beta estimates and the LD among SNPs; as such, it is statistically more powerful than other MR approaches [13].

The GSMR algorithm examines the contribution of exposure on outcome by extracting summary association statistics (Beta, SE) for SNPs included in the instrument from the input files. It then generates a scatter plot by contrasting effect sizes (betas) of SNPs on exposure with their corresponding effect sizes on outcome and calculates the slope $(\beta)$ of the line of best fit and variance around it using generalized least squares regression. In this context, a significant $+\beta$ indicates subjects that are genetically susceptible to higher levels of exposure (e.g., methylation at a site), tend to have higher risk of outcome (e.g., obesity).

To investigate the possibility of reverse causation or the scenario that a change in the methylation level at a site is merely the consequence of gaining weight (BMI $\rightarrow$ Methylation site), we selected methylation-BMI pairs with $p<5 \times 10^{-8}$ from our MR analysis and swapped the places of exposure and outcome. Namely, we set BMI as the exposure and the methylation probe as the outcome, and then, we re-performed the MR test and excluded any methylation-BMI pairs with significant evidence of reverse causation $(p<0.05)$.

\section{Results}

Through our analysis pipeline, we integrated mQTL association summary statistics with GWAS summary statistics for BMI to identify the methylation sites that contribute to the risk of obesity. We identified multiple sites that are causally associated with BMI (Table S1). We replicated (Table S2) these associations using data from Hannon et al. [14,15]. Next, we integrated the transcriptome data from the eQTLGen consortium [16] to investigate the molecular mechanism whereby these sites impact obesity. We review the main findings below.

\subsection{CCNL1 Locus}

Co-localization analysis revealed mQTLs for cg21178254 methylation site upstream of CCNL1 gene co-localize (Table S1) with obesity risk SNPs $\left(\mathrm{P}_{\mathrm{SMR}}=7 \times 10^{-10}, \mathrm{P}_{\mathrm{HEIDI}}=0.5\right)$. The top SNP rs62274156-T in this region was associated with increased BMI $(\mathrm{B}=0.01$, $\left.p=3 \times 10^{-10}\right)$ and lower methylation at the cg21178254 site $\left(B=-0.96, p=5 \times 10^{-182}\right)$. Consistently, the MR analysis revealed that lower methylation at this site contributes to a greater risk of obesity $\left(\mathrm{B}=-0.02, p=1.2 \times 10^{-9}\right.$, Table $\left.\mathrm{S} 1\right)$. 
Next, we investigated whether methylation at the cg21178254 site impacts the expression of nearby genes by performing a MR analysis. Regional association plots for $\mathrm{mQTLs}$ of cg21178254 and eQTLs of the CCNL1 gene were found to overlap (Figure 2A). The MR analysis revealed that as this site becomes methylated, the expression of the CCNL1 gene decreases $\left(\beta=-0.4, p=5 \times 10^{-143}\right.$; Figure $\left.2 \mathrm{~B}\right)$ and this contributes to BMI $(\beta=0.04$, $p=2 \times 10^{-10} ;$ Figure $2 \mathrm{~B}$ ). The expression of no other gene within the $3 \mathrm{q} 25$ chromosome band was affected by methylation of the cg21178254 site. As such, it appears methylation at this site contributes to obesity by changing the expression of CCNL1.

A

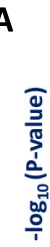
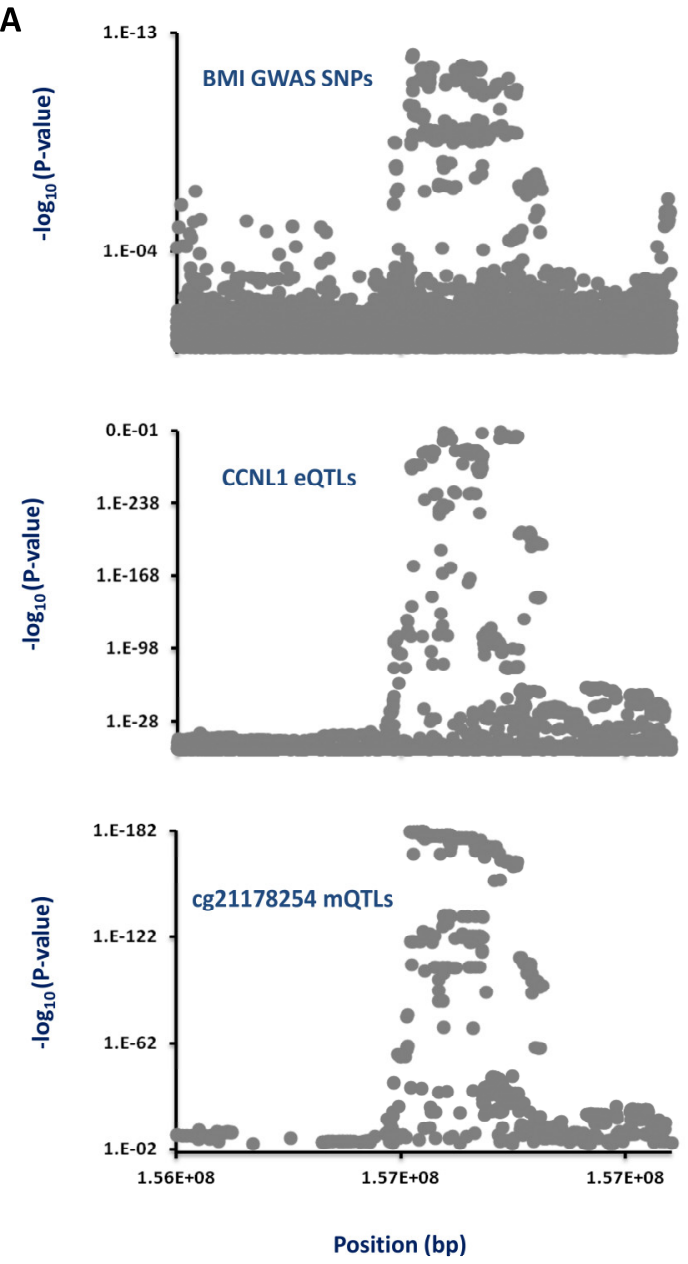

B
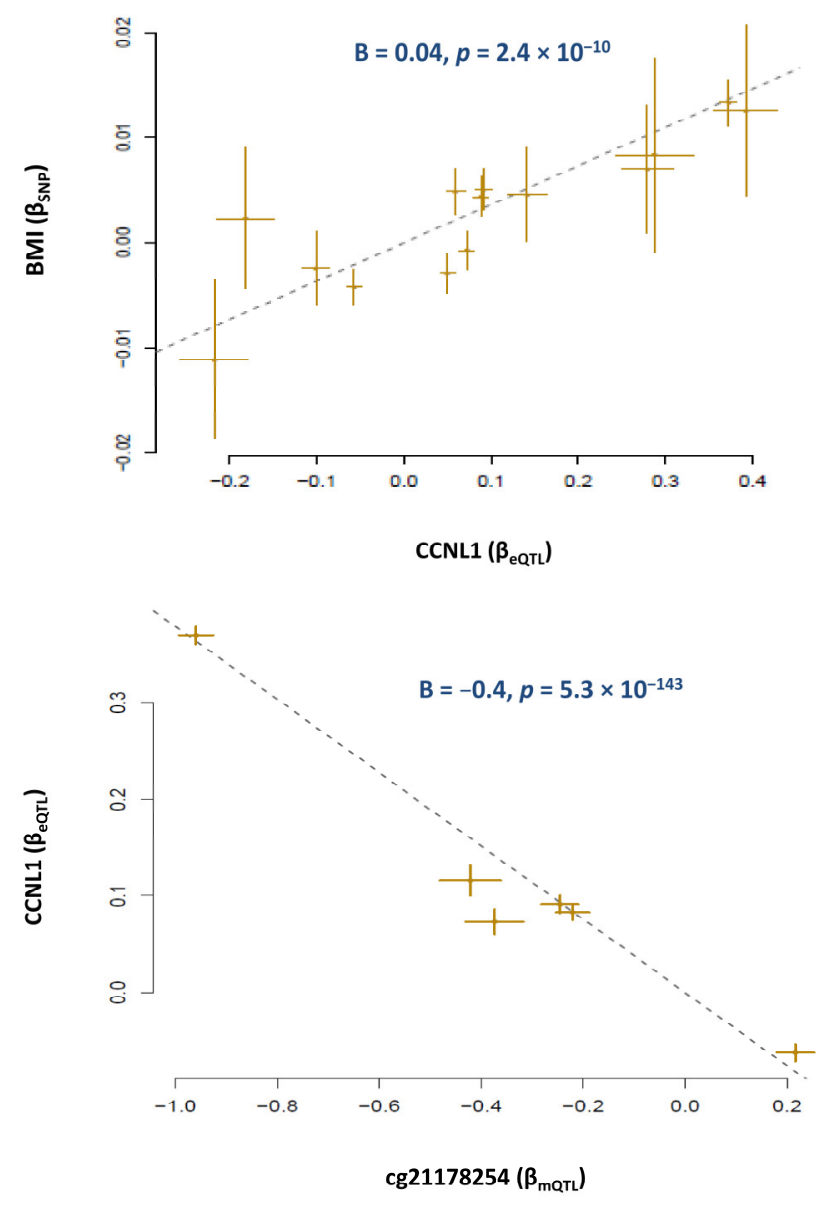

Figure 2. Mechanism whereby the cg20892840 site upstream of CCNL1 impacts obesity. (A) We found that regional association plots for mQTLs of cg20892840, eQTLs of CCNL1 and risk SNPs of obesity co-localize. (B) MR analysis then revealed that, as cg20892840 becomes hypomethylated, the expression of CCNL1 increases, and this contributes to a higher BMI. Each point represents a SNP; the $\mathrm{x}$-value of a SNP is its $\beta$ effect size on the exposure, and the horizontal error bar represents the standard error around the $\beta$. The $y$-value of the SNP is its $\beta$ effect size on the outcome, and the vertical error bar represents the standard error around its $\beta$. The dashed line represents the line of best fit (a line with the intercept of 0 and the slope of $\beta$ from the MR test).

\subsection{SLC5A11 Locus}

We found mQTLs for the $\mathrm{cg} 06028605$ site within the SLC5A11 gene overlap with obesity risk SNPs $\left(\mathrm{P}_{\mathrm{SMR}}=2.6 \times 10^{-10}, \mathrm{P}_{\mathrm{HEIDI}}=0.3\right)$. The top SNP rs34172679-T in this region was associated with a lower $\mathrm{BMI}\left(\mathrm{B}=-0.02, p=2 \times 10^{-10}\right)$ but higher methylation at the cg06028605 site $\left(\mathrm{B}=1.1, p=1 \times 10^{-209}\right)$. Consistently, MR analysis revealed that lower methylation at this site contributes to a higher BMI $\left(B=-0.01, p=3.3 \times 10^{-9}\right.$, Table S1). 
The regional association plots for mQTLs of the cg06028605 site, eQTLs of SLC5A11 and BMI risk SNPs overlap (Figure $3 \mathrm{~A}$ ). MR analysis revealed that this site contributes to the risk of obesity by changing the expression of the SLC5A11 gene. We found that, as this site becomes methylated, the expression of SLC5A11 increases $\left(\mathrm{B}=0.5, p=3.6 \times 10^{-194}\right.$, Figure $3 \mathrm{~B})$ and this contributes to a lower BMI $\left(B=-0.03, p=2.8 \times 10^{-11}\right.$, Figure $\left.3 \mathrm{~B}\right)$.

A
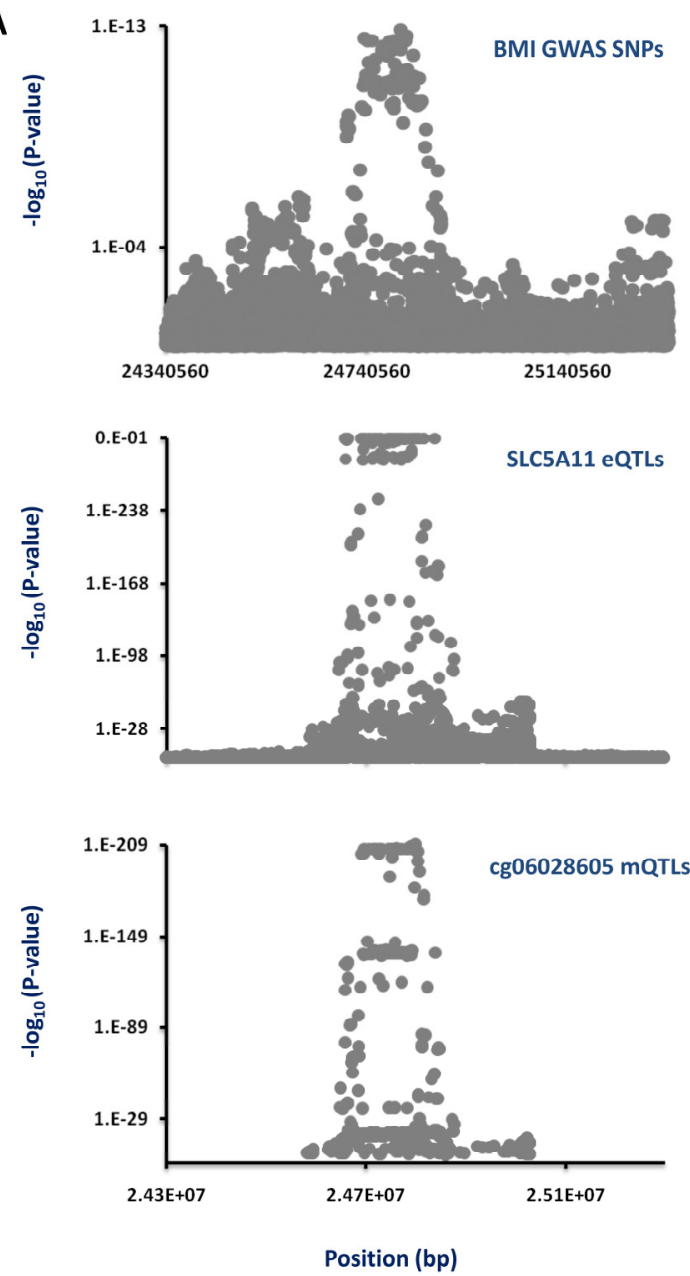

B
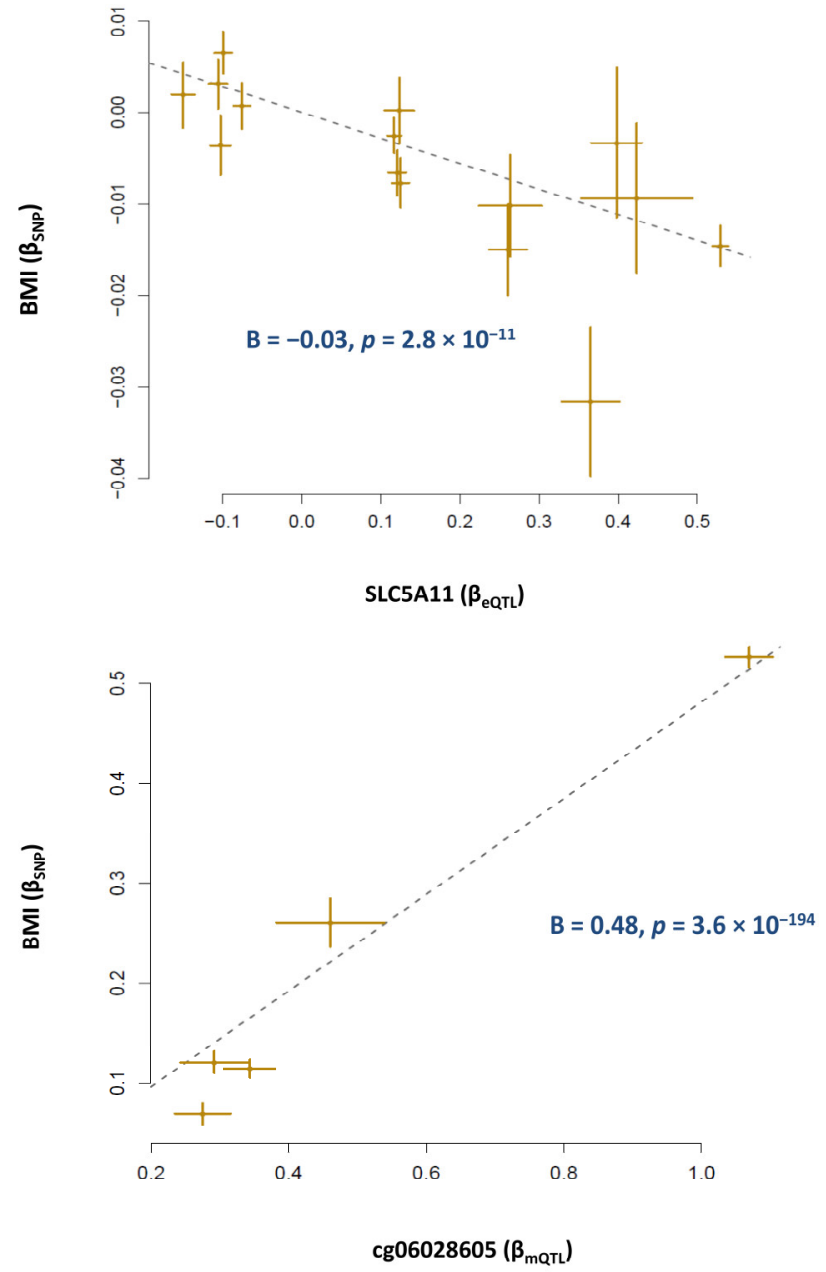

Figure 3. Mechanism whereby the cg06028605 site inside SLC5A11 impacts obesity. (A) We found that the regional association plots for mQTLs of cg06028605, eQTLs of SLC5A11 and risk SNPs of obesity co-localize. (B) MR analysis then revealed that, as the cg06028605 site becomes hypermethylated, the expression of SLC5A11 increases, and this contributes to a lower BMI. Each point on the MR plots represents a SNP; the $x$-value of a SNP is its $\beta$ effect size on the exposure, and the horizontal error bar represents the standard error around the $\beta$. The $y$-value of the SNP is its $\beta$ effect size on the outcome, and the vertical error bar represents the standard error around its $\beta$. The dashed line represents the line of best fit (a line with the intercept of 0 and the slope of $\beta$ from the MR test).

\subsection{MAST3 Locus}

Co-localization and subsequent MR analysis revealed that the cg02814054 methylation site within MAST3 contributes to obesity. We noted that the regional association plots for cg02814054 mQTLs, MAST3 eQTLs and BMI risk SNPs overlap (Figure 4A). The top mQTL, rs4808745-T, within this region was associated with higher methylation ( $\mathrm{B}=0.96$, $\left.p=1.4 \times 10^{-200}\right)$, lower expression of MAST3 $\left(\mathrm{B}=-0.32, p=2.7 \times 10^{-269}\right)$ and a higher $\mathrm{BMI}\left(\mathrm{B}=0.02, p=7 \times 10^{-14}\right.$, Table S1). Concordantly, MR analysis revealed that, as this site becomes methylated, the expression of MAST3 decreases $\left(\mathrm{B}=-0.2, p=1.6 \times 10^{-69}\right.$, Figure $4 \mathrm{~B})$ and the risk of obesity increases $\left(B=-0.06, p=6.9 \times 10^{-14}\right.$, Figure $\left.4 B\right)$. 
A
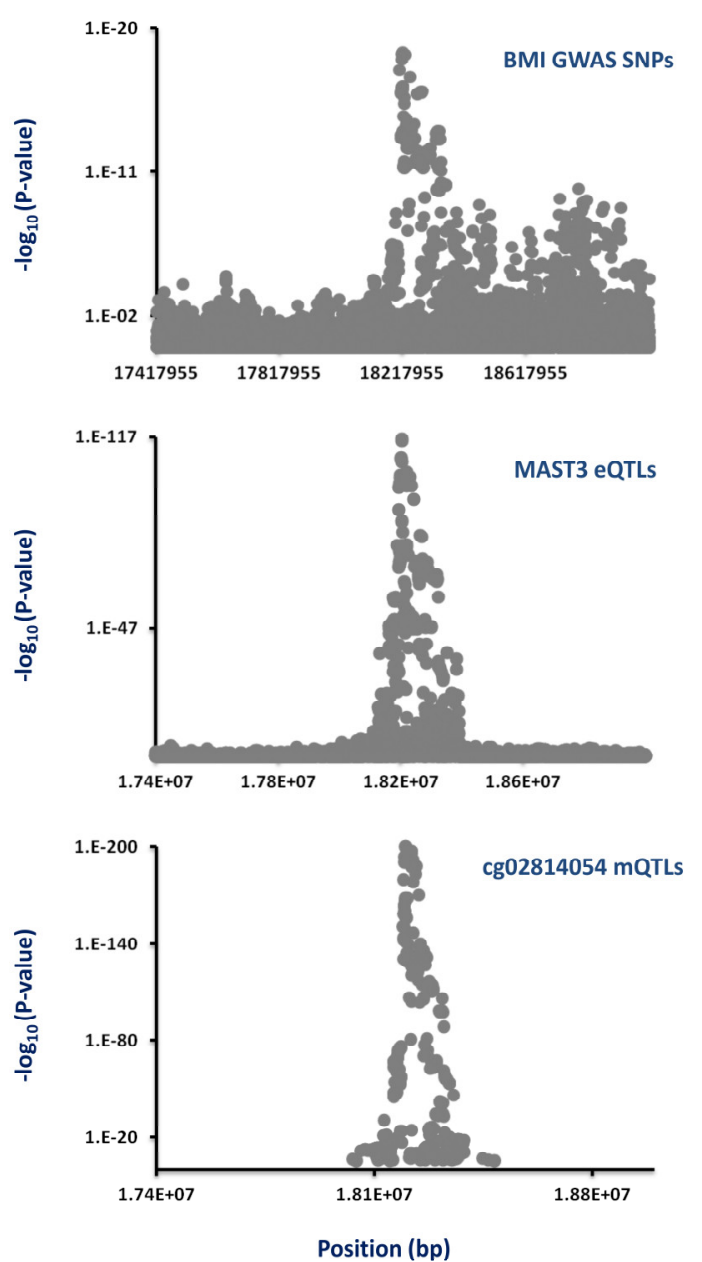
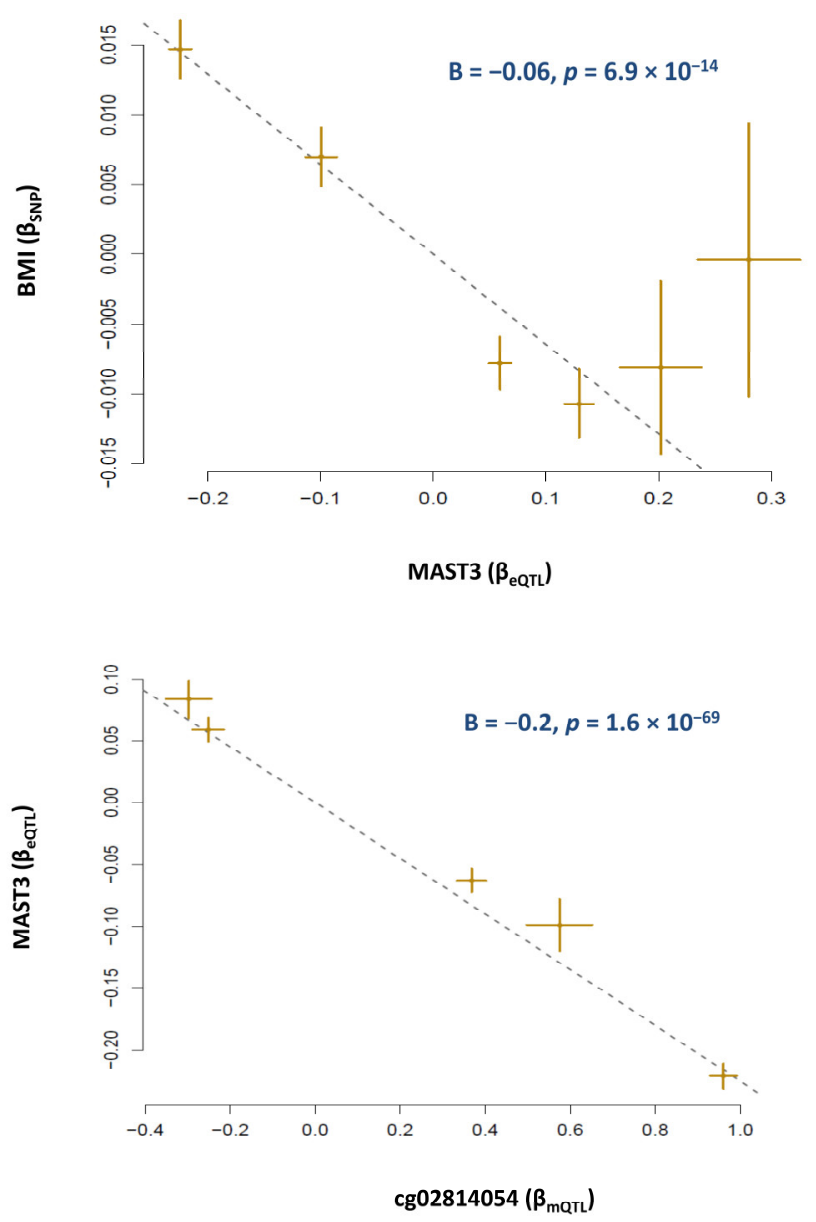

Figure 4. Mechanism whereby the cg02814054 site inside MAST3 impacts obesity. (A) We found that the regional association plots for mQTLs of cg02814054, eQTLs of MAST3 and risk SNPs of obesity co-localize. (B) MR analysis then revealed that, as the cg02814054 site becomes hypermethylated, the expression of MAST3 decreases, and this contributes to a higher BMI. Each point on MR plots represents a SNP; the $\mathrm{x}$-value of a SNP is its $\beta$ effect size on the exposure, and the horizontal error bar represents the standard error around the $\beta$. The $y$-value of the SNP is its $\beta$ effect size on the outcome, and the vertical error bar represents the standard error around its $\beta$. The dashed line represents the line of best fit (a line with the intercept of 0 and the slope of $\beta$ from the MR test).

\subsection{Rare Variants in $2 p 23.3$}

Finally, we examined whether rare obesity SNPs exert their effect through an epigenetic site. For this purpose, we obtained the list of rare variants for obesity from the ClinVar database [17] (Table S3) and matched their position with the position of mQTLs. We noted that rare SNPs within the 2p23.3 chromosome band overlap with mQTLs (blue circles) for a methylation site (cg01884057) within this locus (Figure 5A). MR analysis revealed the higher methylation at this site was associated with a higher $\mathrm{BMI}\left(\mathrm{B}=0.6, p=1.9 \times 10^{-60}\right.$, Figure $5 \mathrm{~B})$. We confirmed this finding using data from a second study as well $(\mathrm{B}=0.03$, $p=1.8 \times 10^{-61}$, Table 1). Higher methylation at this site was associated with lower expression of POMC $\left(\mathrm{B}=-0.11, p=1.75 \times 10^{-37}\right), A D C Y 3\left(\mathrm{~B}=-0.08, p=4.3 \times 10^{-20}\right)$ and $\operatorname{DNAJC27}\left(\mathrm{B}=-0.1, p=5.8 \times 10^{-30}\right.$, Table 1). Furthermore, the lower expression of these genes was associated with a higher risk of obesity (Table 1), consistent with the established role of these genes in obesity. Thus, rare variants within this locus impact obesity by making this site susceptible to methylation, which consequently attenuates the expression of POMC, ADCY 3 and DNAJC27. 
A

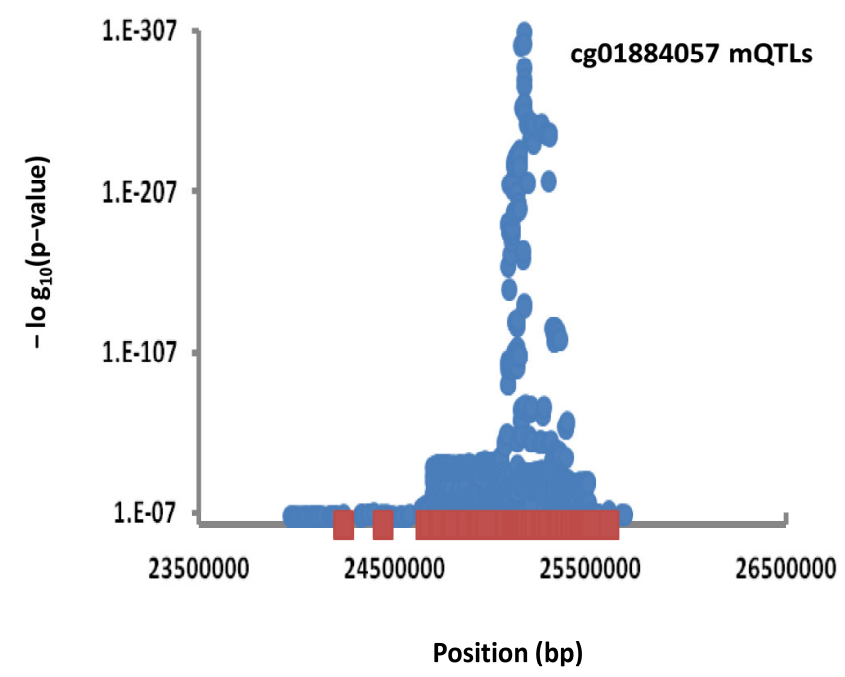

B

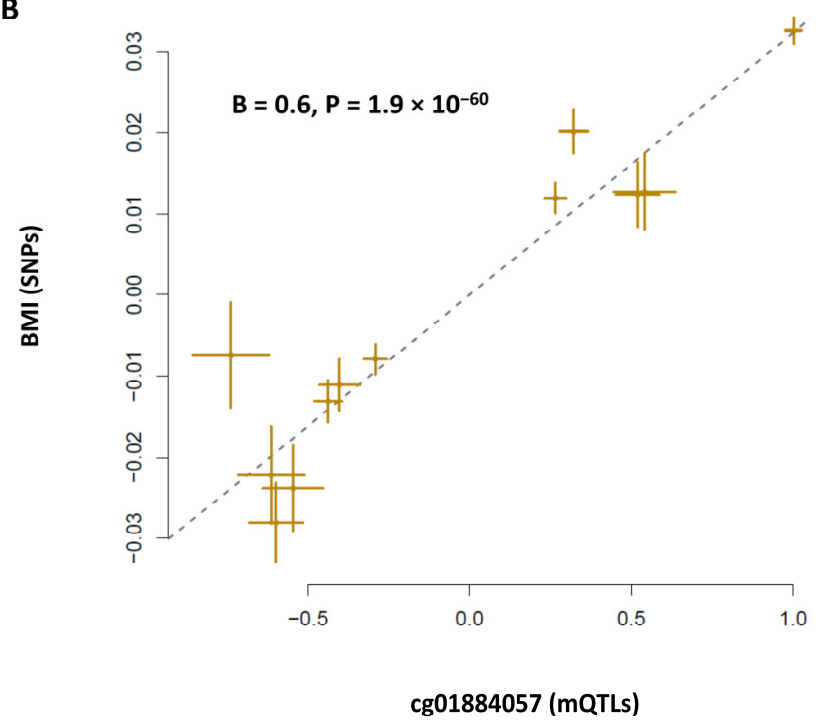

Figure 5. Understanding the function of rare variants using the available knowledge on nearby common SNPs. In this example, we noted that (A) rare variants (red squares) within the 2p23.3 locus overlap with mQTLs (blue circles) for a methylation site (cg01884057) within this locus. (B) MR analysis revealed that higher methylation at this site was associated with a higher BMI. Methylation at this site was also associated with lower expression of POMC, ADCY3 and DNAJC27 (Table 1). Therefore, rare variants within this locus impact obesity by making this site more inclined to methylation, which consequently lowers the expression of POMC, ADCY3 and DNAJC27. Each point on MR plots represents a SNP; the x-value of a SNP is its $\beta$ effect size on the exposure, and the horizontal error bar represents the standard error around the $\beta$. The $y$-value of the SNP is its $\beta$ effect size on the outcome, and the vertical error bar represents the standard error around its $\beta$. The dashed line represents the line of best fit (a line with the intercept of 0 and the slope of $\beta$ from the MR test).

Table 1. Summary association statistics for the mechanism whereby the cg01884057 site contributes to obesity.

\begin{tabular}{|c|c|c|c|c|c|c|c|}
\hline Biomarker & PMID & Oucome & PMID & Beta & SE & $\mathbf{P}$ & NSNP \\
\hline $\operatorname{cg} 01884057$ & 30514905 & $P O M C$ expression & bioRxiv 447367 & -0.11 & 0.01 & $1.7 \times 10^{-37}$ & 5 \\
\hline $\operatorname{cg} 01884057$ & 30401456 & POMC expression & bioRxiv 447367 & -1.90 & 0.15 & $3.6 \times 10^{-37}$ & 4 \\
\hline POMC expression & bioRxiv 447367 & BMI & 30239722 & -0.03 & 0.01 & $1.5 \times 10^{-10}$ & 13 \\
\hline $\operatorname{cg} 01884057$ & 30514905 & $A D C Y 3$ expression & bioRxiv 447367 & -0.08 & 0.01 & $4.3 \times 10^{-20}$ & 12 \\
\hline $\operatorname{cg} 01884057$ & 30401456 & $A D C Y 3$ expression & bioRxiv 447367 & -1.59 & 0.16 & $5.5 \times 10^{-24}$ & 5 \\
\hline$A D C Y 3$ expression & bioRxiv 447367 & BMI & 30239722 & -0.06 & 0.01 & $2.2 \times 10^{-10}$ & 5 \\
\hline $\operatorname{cg} 01884057$ & 30514905 & DNAJC27 expression & bioRxiv 447367 & -0.10 & 0.01 & $5.8 \times 10^{-30}$ & 6 \\
\hline $\operatorname{cg} 01884057$ & 30401456 & DNAJC27 expression & bioRxiv 447367 & -1.56 & 0.14 & $2.3 \times 10^{-27}$ & 4 \\
\hline DNAJC27 expression & bioRxiv 447367 & BMI & 30239722 & -0.05 & 0.01 & $2.7 \times 10^{-10}$ & 7 \\
\hline
\end{tabular}

\section{Discussion}

Here, we performed a genome-wide search for methylation sites that contribute to obesity. Through a discovery and replication stage, we identified seven sites that are causally associated with the risk of obesity. Epigenetic sites are genomic regions that undergo chemical modifications in response to environmental factors; however, the underlying sequence of the DNA also has a role. Namely, some combination of alleles makes a site more susceptible to epigenetic modification than the others. Therefore, our results should be considered in this context.

We integrated the transcriptome data from the eQTLGen consortium [16] to investigate the likely mechanism whereby the methylation sites impact obesity. We found that lower methylation at the cg21178254 site upstream of CCNL1 contributes to obesity by increasing the expression of this gene. We also examined the data from the PhenomeXcan database [18], which is a comprehensive repertoire of gene expression-trait associations. 
Results from this database also indicated that a higher expression of this gene is associated with a higher risk of BMI $\left(p=1.4 \times 10^{-6}\right)$. Previous studies found that SNPs upstream of CCNL1 are associated with leptin levels [19] and birth weight [20]. We also noted that a change in expression of this gene is associated $\left(p<1 \times 10^{-10}\right)$ with comparative height at age 10 as well as standing height; therefore, this gene may impact anthropometric traits including BMI by a change in transcriptional activity early in life. This is consistent with the function of this gene, which is a cyclin-dependent kinase and is involved in regulating transcription and mRNA processing.

We noted that the lower methylation at the cg06028605 site in the SLC5A11 locus contributes to obesity by decreasing the expression of this gene. Data from PhenomeXcan indicated that the lower expression of this gene is associated with a higher BMI $\left(p=1 \times 10^{-7}\right)$. SLC5A11 is involved in the transport of glucose and other sugars, bile salts, organic acids, metal ions and amine compounds. The gene is known to have a regulatory impact on appetite. Food deprivation is reported to increase the expression of SLC5A11 and the excitability of SLC5A11-expressing EB R4 neurons [21]. Ugrankar et al. [22] reported that SLC5A11 is involved in glucose-regulated feeding and may function downstream of insulin signaling on the pathway of glucose sensing.

We found that the higher methylation at the cg02814054 site within MAST3 contributes to obesity by lowering the expression of this gene. Data from PhenomeXcan also revealed that the lower expression of this gene contributes to a higher BMI $\left(p=1 \times 10^{-8}\right)$. MAST3 is a microtubule-associated serine/threonine-protein kinase. The gene appears to have diverse activities and is implicated in different diseases. Therefore, the molecular path whereby a change in expression of this gene contributes to obesity remains elusive. Data from PhenomeXcan (based on GTEX and UKBB data) indicated that MAST3 has a higher expression in people with a college or a university degree $\left(p=7.2 \times 10^{-7}\right)$. We also confirmed this effect through MR analysis $\left(B=0.03, p=5.8 \times 10^{-9}\right.$, using data from eQTLGen and UKBB). Therefore, the mechanism whereby a change in expression of this gene contributes to obesity may be through the neural paths. This is reasonable considering that CNS contributes to obesity and a number of studies have documented the neural function of this gene $[23,24]$.

Over the past decades, GWAS studies have identified SNPs that contribute to phenotopic variability, and high-throughput studies have identified SNPs underlying functional elements. With the availability of data from these studies, and analytical tools that rely on summary association statistics, now is the time to integrate findings from these studies and catalog the association of functional elements with the phenome. In this work and previously $[8,9,25]$, we showed such studies are feasible through a SNP-based multiomics approach. Furthermore, in this study, we show that such an approach can be used to understand the function of rare genomic variants (Figure 5). Although advances in sequencing technology allow us to capture rare variants efficiently, it often remains elusive which variant has a causal impact on the examined phenotype. We demonstrate that, by leveraging the abundance of knowledge available at nearby common SNPs, it is possible to investigate the mechanism whereby a rare SNP impacts a phenotype. Such an approach also provides the possibility to identify the causal rare SNP in a list of candidate SNPs.

This study is notable considering that it combines several sets of publicly available data to reveal new findings; therefore, it demonstrates the value of data sharing by researchers and supports this practice. Compared to traditional EWAS studies, this study is unique because it exploits the power of MR to control for confounding and reverse causation; as such, it provides a framework for performing omics-wide association studies of phenotypes. Our study has a number of limitations; in this study, we could not reveal any functional insight for a number of the identified sites (Table S1). Therefore, future studies require integrating a more diverse and comprehensive set of data in order to investigate the mechanism whereby a functional element impacts a phenotype. Another limitation of this study is that we could not investigate trans-regulatory effects since the majority of QTL studies (including those examined in this study) report their findings for cis-acting QTLs 
(e.g., eQTLs within a gene's own regulatory region). Reporting QTLs with trans-regulatory effects (e.g., eQTLs located in a different chromosome than their gene) is highly valuable because they can provide novel insights into the molecular pathway whereby a biomarker exerts its effect.

In summary, in this study, we integrated several sets of data to identify epigenetic sites that contribute to obesity. For a number of these sites, we revealed the mechanism through which they impact obesity. Finally, we showed that, by leveraging the knowledge of common SNPs, it is possible to prioritize rare variants and investigate the mechanism whereby a causal variant impacts a phenotype.

Supplementary Materials: The following are available online at https:/ /www.mdpi.com/article/10 $.3390 /$ nu13061984/s1, Table S1. Summary association statistics for epigenetic sites that are causally associated with BMI; Table S2. Replication of probe-BMI causal associations using data from additional studies; Table S3. List of rare variants for obesity obtained from the ClinVar database; Table S4. Summary association statistics that extracted from the previous studies and used in the current study to examine the causal association between change in DNA methylation and BMI using MR; Table S5. Summary association statistics that extracted from the previous studies and used in the current study to examine the causal association between change in DNA methylation and gene expression using MR.

Author Contributions: Conceptualization, M.N., R.M. and R.D.; formal analysis, M.N.; investigation, S.R.; resources, R.M. and R.D.; writing—original draft preparation, M.N.; writing-review and editing, R.M. and S.R. All authors have read and agreed to the published version of the manuscript.

Funding: Supported by the Canadian Institutes of Health Research \# FDN-154308 (RM).

Institutional Review Board Statement: Not applicable, this study was done using publicly available data.

Informed Consent Statement: Not applicable.

Data Availability Statement: Summary association statistics to replicate our findings are provided in the Supplementary Tables S4 and S5. Samples scripts and input files to perform a SNP-based multiomics analysis are available from: https://github.com/mnikpay/Multiomics-MR-scripts.git (accessed on 8 June 2021).

Acknowledgments: This research was enabled in part by computational resources and support provided by the Compute Ontario (https: / /www.computeontario.ca/, accessed on 8 June 2021) and the Compute Canada (https:/ /www.computecanada.ca, accessed on 8 June 2021). We thank the reviewers and editors for their thoughtful comments and efforts toward improving this manuscript.

Conflicts of Interest: The authors declare no conflict of interest.

\section{References}

1. Ling, C.; Rönn, T. Epigenetics in human obesity and type 2 diabetes. Cell Metab. 2019, 29, 1028-1044. [CrossRef]

2. Low, F.M.; Gluckman, P.D.; Hanson, M.A. Epigenetic and Developmental Basis of Risk of Obesity and Metabolic Disease. In Cellular Endocrinology in Health and Disease, 2nd ed.; Ulloa-Aguirre, A., Tao, Y.-X., Eds.; Academic Press: Boston, MA, USA, 2021; Chapter 14, pp. 289-313.

3. Xu, X.; Su, S.; Barnes, V.A.; De Miguel, C.; Pollock, J.; Ownby, D.; Shi, H.; Zhu, H.; Snieder, H.; Wang, X. A genome-wide methylation study on obesity: Differential variability and differential methylation. Epigenetics 2013, 8, 522-533. [CrossRef]

4. Wahl, S.; Drong, A.; Lehne, B.; Loh, M.; Scott, W.R.; Kunze, S.; Tsai, P.-C.; Ried, J.S.; Zhang, W.; Yang, Y. Epigenome-wide association study of body mass index, and the adverse outcomes of adiposity. Nature 2017, 541, 81-86. [CrossRef]

5. Koh, I.-U.; Choi, N.-H.; Lee, K.; Yu, H.-Y.; Yun, J.H.; Kong, J.-H.; Kim, H.J.; Lee, S.; Kim, S.C.; Kim, B.-J.; et al. Obesity susceptible novel DNA methylation marker on regulatory region of inflammation gene: Results from the Korea Epigenome Study (KES). BMJ Open Diabetes Res. Care 2020, 8, e001338. [CrossRef]

6. Mendelson, M.M.; Marioni, R.E.; Joehanes, R.; Liu, C.; Hedman, Å.K.; Aslibekyan, S.; Demerath, E.W.; Guan, W.; Zhi, D.; Yao, C. Association of body mass index with DNA methylation and gene expression in blood cells and relations to cardiometabolic disease: A Mendelian randomization approach. PLoS Med. 2017, 14, e1002215. [CrossRef]

7. Davies, N.M.; Holmes, M.V.; Davey Smith, G. Reading Mendelian randomisation studies: A guide, glossary, and checklist for clinicians. BMJ 2018, 362, k601. [CrossRef] 
8. Nikpay, M.; McPherson, R. Convergence of biomarkers and risk factor trait loci of coronary artery disease at 3p21.31 and HLA region. npj Genom. Med. 2021, 6, 12. [CrossRef] [PubMed]

9. Nikpay, M.; Soubeyrand, S.; Tahmasbi, R.; McPherson, R. Multiomics Screening Identifies Molecular Biomarkers Causally Associated with the Risk of Coronary Artery Disease. Circ. Genom. Precis. Med. 2020, 13, e002876. [CrossRef]

10. McRae, A.F.; Marioni, R.E.; Shah, S.; Yang, J.; Powell, J.E.; Harris, S.E.; Gibson, J.; Henders, A.K.; Bowdler, L.; Painter, J.N.; et al. Identification of 55,000 Replicated DNA Methylation QTL. Sci. Rep. 2018, 8, 17605. [CrossRef] [PubMed]

11. Pulit, S.L.; Stoneman, C.; Morris, A.P.; Wood, A.R.; Glastonbury, C.A.; Tyrrell, J.; Yengo, L.; Ferreira, T.; Marouli, E.; Ji, Y.; et al. Meta-analysis of genome-wide association studies for body fat distribution in 694649 individuals of European ancestry. Hum. Mol. Genet. 2018, 28, 166-174. [CrossRef] [PubMed]

12. Zhu, Z.; Zhang, F.; Hu, H.; Bakshi, A.; Robinson, M.R.; Powell, J.E.; Montgomery, G.W.; Goddard, M.E.; Wray, N.R.; Visscher, P.M.; et al. Integration of summary data from GWAS and eQTL studies predicts complex trait gene targets. Nat. Genet. 2016, 48, 481-487. [CrossRef] [PubMed]

13. Zhu, Z.; Zheng, Z.; Zhang, F.; Wu, Y.; Trzaskowski, M.; Maier, R.; Robinson, M.R.; McGrath, J.J.; Visscher, P.M.; Wray, N.R.; et al. Causal associations between risk factors and common diseases inferred from GWAS summary data. Nat. Commun. 2018, 9, 224. [CrossRef] [PubMed]

14. Hannon, E.; Gorrie-Stone, T.J.; Smart, M.C.; Burrage, J.; Hughes, A.; Bao, Y.; Kumari, M.; Schalkwyk, L.C.; Mill, J. Leveraging DNA-Methylation Quantitative-Trait Loci to Characterize the Relationship between Methylomic Variation, Gene Expression, and Complex Traits. Am. J. Hum. Genet. 2018, 103, 654-665. [CrossRef] [PubMed]

15. Hannon, E.; Dempster, E.; Viana, J.; Burrage, J.; Smith, A.R.; Macdonald, R.; St Clair, D.; Mustard, C.; Breen, G.; Therman, S.; et al. An integrated genetic-epigenetic analysis of schizophrenia: Evidence for co-localization of genetic associations and differential DNA methylation. Genome Biol. 2016, 17, 176. [CrossRef]

16. Võsa, U.; Claringbould, A.; Westra, H.-J.; Bonder, M.J.; Deelen, P.; Zeng, B.; Kirsten, H.; Saha, A.; Kreuzhuber, R.; Kasela, S.; et al. Unraveling the polygenic architecture of complex traits using blood eQTL metaanalysis. bioRxiv 2018, 447367. [CrossRef]

17. Landrum, M.J.; Chitipiralla, S.; Brown, G.R.; Chen, C.; Gu, B.; Hart, J.; Hoffman, D.; Jang, W.; Kaur, K.; Liu, C. ClinVar: Improvements to accessing data. Nucleic Acids Res. 2020, 48, D835-D844. [CrossRef]

18. Pividori, M.; Rajagopal, P.S.; Barbeira, A.; Liang, Y.; Melia, O.; Bastarache, L.; Park, Y.; Consortium, G.; Wen, X.; Im, H.K. PhenomeXcan: Mapping the genome to the phenome through the transcriptome. Sci. Adv. 2020, 6, eaba2083. [CrossRef] [PubMed]

19. Kilpeläinen, T.O.; Carli, J.F.M.; Skowronski, A.A.; Sun, Q.; Kriebel, J.; Feitosa, M.F.; Hedman, Å.K.; Drong, A.W.; Hayes, J.E.; Zhao, J. Genome-wide meta-analysis uncovers novel loci influencing circulating leptin levels. Nat. Commun. 2016, 7, 1-14. [CrossRef]

20. Horikoshi, M.; Yaghootkar, H.; Mook-Kanamori, D.O.; Sovio, U.; Taal, H.R.; Hennig, B.J.; Bradfield, J.P.; Pourcain, B.S.; Evans, D.M.; Charoen, P. New loci associated with birth weight identify genetic links between intrauterine growth and adult height and metabolism. Nat. Genet. 2013, 45, 76-82. [CrossRef]

21. Park, J.-Y.; Dus, M.; Kim, S.; Abu, F.; Kanai Makoto, I.; Rudy, B.; Suh Greg, S.B. Drosophila SLC5A11 Mediates Hunger by Regulating K+ Channel Activity. Curr. Biol. 2016, 26, 1965-1974. [CrossRef]

22. Ugrankar, R.; Theodoropoulos, P.; Akdemir, F.; Henne, W.M.; Graff, J.M. Circulating glucose levels inversely correlate with Drosophila larval feeding through insulin signaling and SLC5A11. Commun. Biol. 2018, 1, 110. [CrossRef]

23. Garland, P.; Quraishe, S.; French, P.; O'Connor, V. Expression of the MAST family of serine/threonine kinases. Brain Res. 2008, 1195, 12-19. [CrossRef] [PubMed]

24. Spinelli, E.; Christensen, K.R.; Bryant, E.; Schneider, A.; Rakotomamonjy, J.; Muir, A.M.; Giannelli, J.; Littlejohn, R.O.; Roeder, E.R.; Schmidt, B.; et al. Pathogenic MAST3 variants in the STK domain are associated with epilepsy. bioRxiv 2021. [CrossRef]

25. Nikpay, M.; Beehler, K.; Valsesia, A.; Hager, J.; Harper, M.-E.; Dent, R.; McPherson, R. Genome-wide identification of circulatingmiRNA expression quantitative trait loci reveals the role of several miRNAs in the regulation of cardiometabolic phenotypes. Cardiovasc. Res. 2019, 115, 1629-1645. [CrossRef] [PubMed] 